\title{
45. Jahreskongress der Deutschen Gesellschaft für Transfusionsmedizin und Immunhämatologie (DGTI)
}

\author{
11.-14. September 2012, Graz
}

\author{
Die Abstracts waren nicht den Oral oder Poster Sessions des 45. Jahreskongresses der \\ Deutschen Gesellschaft für Transfusionsmedizin und Immunhämatologie (DGTI) zugeordnet \\ und daher nicht im Abstractband publiziert worden.
}

\section{An Electronic Management System for Quality Management}

Mueller-Kuller $T^{1}$, , Spyrantis $A .{ }^{1}$, Findhammer $S .{ }^{1}$, Knels $R^{2}{ }^{2}$ Karl $A^{3}{ }^{3}$, Sireis $W{ }^{1}$

${ }^{1}$ DRK Blutspendedienst Baden-Württemberg - Hessen gGmbH, Qualitätsmanagement, Frankfurt am Main, Deutschland

${ }^{2}$ DRK Blutspendedienst Ost gGmbH, Institutsleiter Cottbus, Cottbus, Deutschland

${ }^{3}$ DRK Blutspendedienst Ost gGmbH, Qualitätsmanagement, Plauen, Deutschland

Document management is both essential and challenging for quality management. In order to enhance traceability and reduce paper copies we implemented an electronic system for document management and archiving. We decided for the "Saperion" document archiving system and defined customized workflows for SOP creation / revision, risk management and interna audits. Among the most important features of this implementation are the accelerated processing time and optimized document control over all of ou participating institutes. This system is accessible to all employees, to which accessibility rights and task related interfaces are specifically adapted to their duties.

Furthermore we also use this system to archive filled-in quality documents Therefore every document has a distinct number assigned to it. Quality documents have a barcode representing their specific number and the document index providing all important administrative information. Archiving of these quality documents is facilitated by the information provided through the document index field, once the barcode is read. This enables large scale archiving and reduce archive storage space.

Processes that include third parties, such as regulatory agencies are processed in the same way as before, but the outcome is submitted in our Saperion database. Each file is easily retrievable through a user friendly search function. Such an example includes our hemovigilance module which contains interfaces for look-back cases and adverse reactions.

Throughout the entire workflow or input processes, every step is documented and every change is archived as an internal revision, thus ensuring a complete traceability of document and data management throughout all steps. We are constantly working on further introducing and establishing new modules on this system in order to replace existing paper form processes and to easily provide access to relevant data on demand.

\author{
IT-Validation (GAMP 5) \\ Steinke $W^{1}{ }^{1}$, Bux J. ${ }^{2}$ \\ ${ }^{1}$ DRK Blutspendedienst West gGmbH, Münster, Deutschland \\ ${ }^{2}$ DRK Blutspendedienst West gGmbH, Hagen, Deutschland;
}

Introduction: The Good Automated Manufacturing Practice Supplier Guide for Validation of Automated Systems in Pharmaceutical Manufacture (GAMP) is the standard set of guidelines for manufacturers and users of automated systems in the pharmaceutical industry. Principles and procedures described help to ensure that pharmaceutical products have the required quality.

The Quality management of the German Red Cross Blood Transfusion Service West is using GAMP 5. In this lecture the experiences of using GAMP 5 are described.

Methods: The IT-validation activities of the German Red Cross Blood Transfusion Service West are performed according to the set of principles and procedures of GAMP 5. There are some basic elements needed from the Risk Management to the Validation Report (e.g. Change Control, training of staff). Furthermore, a validated system includes elements such as Annual Product Review or Corrective and Preventive action (CAPA) etc.

The most important aspects of the needs of a validated system are described. Results / Conclusions: IT-validation activities according to GAMP 5 are generating a structured documentation. This documentation is necessary to the manufacturers and users as well as to the authorities.

\section{Blood ID: Bonding of Temperature Monitoring and "Tracking and Tracing"}

Wagner $T^{1}{ }^{1}$, Ockenfuss $U^{2}{ }^{2}$, Speletz $H_{.}{ }^{3}$, Diemer M. ${ }^{3}$, Lanzer $G .{ }^{1}$ ${ }^{1}$ Department of Blood Group Serology and Transfusion Medicine, Medical University Graz, Österreich

${ }^{2}$ Schweizer Electronic AG, Schramberg, Deutschland

${ }^{3}$ Siemens AG Austria, Automation \& Drives, Österreich

${ }^{4}$ MacoPharma International $\mathrm{GmbH}$, Deutschland

Red blood cells (RBCs) are stored at $4 \pm 2{ }^{\circ} \mathrm{C}$ for almost 6 weeks. Once the $\mathrm{RBC}$ unit was released from the blood bank it will not be redeemed by them. Therefore, blood units not used due to various reasons were discarded. To reduce this withdraws (up to $10 \%$ ) different attempts were made to track each single blood unit. In order to get valid temperature data from RBC units, temperature was monitored using an experimental approach with temperature leads as well as a RFID device (SEAGSense). The latter one was developed to enable both 'tracking and tracing' and temperature moni-

\section{KARGER \\ Fax +497614520714 \\ Information@Karger.de}

www.karger.com 
toring of each single RBC unit. Using this device one is able to document all GMP-relevant data and the temperature of each unit and based on these data one can decide about discarding or further use of this unit.

This RFID based solution was developed by an industrial consortium including Siemens AG Austria, Schweizer Electronic AG and MacoPharma together with the blood bank in Graz. This RFID tag is equipped with a battery to enable complete temperature monitoring and should be fixed on each single RBC unit bag prior to whole blood donation and accompany the unit until transfusion or discard (from "vein-to-vein"). Due to the fact that whole blood units have to be centrifuged to allow separation into different blood products, the RFID tag has to stand a centrifugal force of as high as $4000 \mathrm{~g}$. This was in fact the biggest challenge especially with respect to battery function. This problem could be solved so that each RFID tag can be centrifuged as often as 20 times, which will allow multiple use and subsequently reduce costs. The guaranteed expected useful life of the battery is 3 years. The RFID tag is currently equipped with a measuring device and a $13.56 \mathrm{MHz}$ frontend, which is ISO 15693 compatible. When integrated in the local SW program of both the blood bank and the hospital information system a complete "tracking and tracing" including reports to national hemovigilance register is possible.

\section{State of the Art: Data Management and Transfusion Medicine}

Knels $R$.

German Red Cross, Blood Donor Service East, Institute Cottbus, Deutschland

In the last decade new IT solutions changed our daily live. Similar to the introduction of first IT solutions, the penetration of new improvements into health care sector is delayed again. But what is reality today and what are future opportunities? The lecture deals with the introduction of existing solutions as well as older and new IT ideas in Transfusion Medicine.

Topics included:

- rule of internet and social networks for donor loyalty programs

- electronic donor questionnaires

- acceptance analysis and roll out of the new German Red Cross wide implemented RFID based donor ID-card

- data capture with RFID

- electronic recording and archiving of process data

- standard interface solutions and lab automation

- IT-based quality management systems

- IT-validation

- data protection

- patient identification and management support systems

- haemovigilance
For the lecture, the knowledge of the ISBT-and DGTI-Working Parties "Information Technology" will be used. Thus the referent, chairperson of both, thanks all members of both working parties for their activities and likes to invite all interested persons to join the working parties.

\section{Blood Donor Screening by NAT or Serology - Which Strategy Is More Cost Effective?}

Seifried E., Hourfar M.K., Sireis W., Schmidt M.

DRK Blutspendedienst Baden-Württemberg - Hessen gGmbH, Frankfurt am Main, Deutschland

Introduction: Blood Donor Screening is done in many countries by a combination of serology screening and nucleic acid testing (NAT) in order to reduce the diagnostic window period to a minimum and to improve blood safety to the highest level. Cost effectiveness calculations traditionally are based on antibody/antigen screening assays as a first line strategy.

Background: The study presents cost effectiveness and QALY calculations for antibody/antigen screening tests as well as for NAT screening tests under the assumption of four different screening strategies to obtain the most effective donor screening on the highest safety level.

Methods: Epidemiological data of the Blood Donor Service BadenWürttemberg - Hessen were analyzed between 2007 and 2010 for HBV, $\mathrm{HCV}$ and HIV-1. Cost effectiveness as well as calculations for cost per QALY gained were done for four scenarios. A: traditional strategy (serological screening first line); B: window period screening strategy (MP-NAT screening first line); C: biological screening strategy (MPNAT only) and D: combined NAT/serology strategy.

Results: Within the studied period of four years; 1, 6 and 1 MP-NAT only positive blood donations were detected by for $\mathrm{HBV}, \mathrm{HCV}$ and HIV-1, respectively.

Table 1 summarizes the cost per QALY gained for all scenarios.

By changing the calculation model for the window period strategy (MPNAT screening first line) or the biological screening strategy (screening by MP-NAT only) the costs per QALY gained were acceptable for MPNAT for all three pathogens, whereas the cost per QALY gained increased to an unacceptable level higher than $10^{9} €$ for all serology screening assays.

Conclusions: It is recommended that cost efficiency calculations as well as calculations on costs per QALY gained should be done for the combination of NAT and serology screening methods (combined strategy), because both technologies are needed to improve blood safety to the highest level for blood components.

Table 1: Cost per QALY gained

\begin{tabular}{|c|c|c|c|c|c|c|c|c|c|}
\hline $\begin{array}{l}\text { Cost per } \\
\text { QALY gained }(€)\end{array}$ & HBsAg & $\begin{array}{l}\text { HBV MP- } \\
\text { NAT }\end{array}$ & $\begin{array}{l}\text { HBV ID- } \\
\text { NAT }\end{array}$ & Anti-HCV & $\begin{array}{l}\text { HCV MP- } \\
\text { NAT }\end{array}$ & $\begin{array}{l}\text { HCV ID- } \\
\text { NAT }\end{array}$ & $\begin{array}{c}\text { HIV } \\
\text { Combo }\end{array}$ & $\begin{array}{l}\text { HIV-MP- } \\
\text { NAT }\end{array}$ & $\begin{array}{l}\text { HIV ID- } \\
\text { NAT }\end{array}$ \\
\hline A: traditional strategy & 28,670 & $1.88 \mathrm{E}+07$ & $1.94 \mathrm{E}+08$ & 28,249 & $1.67 \mathrm{E}+06$ & $1.33 \mathrm{E}+07$ & 9,390 & 422,535 & 4.37E+07 \\
\hline $\begin{array}{l}\text { B: window period } \\
\text { strategy }\end{array}$ & $1.00 \mathrm{E}+09$ & 59,242 & 856,164 & 190,476 & 38,314 & 574,713 & $1.00 \mathrm{E}+09$ & 17,606 & 264,085 \\
\hline C: biological strategy & $1.00 \mathrm{E}+09$ & 59,242 & 856,164 & $1.00 \mathrm{E}+09$ & 38,314 & 574,713 & $1.00 \mathrm{E}+09$ & 17,606 & 264,085 \\
\hline D: combined strategy & \multicolumn{2}{|c|}{$8.56 \mathrm{E}+04$} & & \multicolumn{2}{|c|}{$4.10 \mathrm{E}+04$} & & \multicolumn{2}{|c|}{$2.64 \mathrm{E}+04$} & \\
\hline
\end{tabular}

The traditional strategy (A) is based on cost efficiency calculations for serology screening methods as a first line. The window period strategy (B) represents calculations for NAT in a first line. Within the study period no samples were HBsAg or HIV-1 antibody only reactive. Therefore costs per QALY gained achieved values higher than $1.0 \mathrm{E}+09 €$. For Anti-HCV 35 samples were confirmed antibody reactive but NAT negative. The biological strategy $(\mathrm{C})$ is based on the assumption that only NAT positive blood donations were infective. Under these conditions costs per QALY gained increased for all serological tests values higher than $1.0 \mathrm{E}+09 €$. The combined strategy (D) covers the real situation that blood donor screening by NAT is on risk of false negative screening results due to mutations within the primer and probe binding region. Therefore blood donor screening should be done in a combination of both screening strategies. 


\section{Human Cytomegalovirus Infections in Blood Donors - an Update}

Ziemann M., Görg S., Hennig H.

University Hospital of Schleswig-Holstein, Institute of Transfusion Medicine, Lübeck, Deutschland

Background: About every second blood donor in Germany is latently infected with Human Cytomegalovirus (CMV), while primary infections occur in about $1 \%$ of seronegative donors per year. Traditionally, window phases with prominent viremia, circulation of latently infected leukocytes and reactivations of latent infections even in healthy donors were thought to characterize CMV infections. Therefore blood donations from latently infected blood donors, and - to a lesser extent - donations during the window phase of primary CMV infections, were hold responsible for transfusion-transmitted CMV infections (TT-CMV).

Methods: Overview about recent developments.

Results / Conclusions: During recent years, new data about CMV infections in blood donors has changed the knowledge about CMV infection in blood donors: Prevalences of window period donations among seronegative donors and reactivations among long-term seropositive donors, as well as the CMV DNA-concentration in whole blood and plasma samples from these donor are low. Primarily seropositive donors, however, show distinctly higher concentrations of CMV DNA in both whole blood and plasma. The prevalence of CMV DNA in these donors depends on the interdonation interval and can reach $80 \%$ or more. Therefore, leukoreduced blood products of seronegative and long-term seropositive blood donors could be used for patients at risk for TT-CMV. Blood products from primarily seropositive donors, however, seem to hold the highest risk of TT-CMV. Avoiding these blood products for immunocompromised patients is easily possible by CMV antibody testing.

\section{Detection of Non-Cell Associated Human Cytomegalovirus in Leukocyte-Reduced Blood Products}

Weber-Schehl M. ${ }^{1}$, Hedges $D .{ }^{1}$, Weinauer $F^{2}$

${ }^{1}$ Blutspendedienst des BRK gGmbH, Molekulare Virusdiagnostik, Wiesentheid, Deutschland

${ }^{2}$ Blutspendedienst des BRK gGmbH, München, Deutschland

Introduction: According to German guidelines [1], the use of leukocytedepleted blood products is an effective strategy of reducing transfusion transmitted human Cytomegalovirus infection. A remaining risk still exists due to free virus. Therefore, we introduced an in-house CMV-NAT for the detection of virus in plasma of blood samples and products. Blood products with higher plasma content, such as platelets, are tested with a more sensitive NAT.

Methods: CMV-NAT is performed in minipools from EDTA plasma. An input volume of 0.1 plasma of each sample is pooled. Detection limit of CMV-NAT screening was determined with $1,000 \mathrm{IU} / \mathrm{ml}$. PCR set-up for testing platelet concentrates, products with higher plasma content, a volume of $1 \mathrm{ml}$ of each tested sample is used, resulting in 10fold sensitivity. Isolation of viral nucleic acids is performed by autoX-System (GFE Blut $\mathrm{mbH}$ ) [2]. DNA samples are analyzed with an in-house real-time PCR system (ABI7500), using published oligonucleotide sequences [3]. In case of PCR positive results, presence of $\operatorname{IgM}$ and $\mathrm{IgG}$ antibodies to $\mathrm{CMV}$ are tested by ELISA.

Results / Conclusions: Screening 351,231 whole blood donations for CMVDNA, from November 2010 to March 2012, identified 14 DNA positive donations $(0.004 \%)$. In the same period of time 23,985 donations for preparation of platelets (pool- and apheresis platelets) were screened for CMV by NAT, of which 9 donations were tested positive $(0.04 \%)$. Higher yield of NAT-positive donations can be explained by higher input volume of analyzed plasma. CMV infection was confirmed by screening for antibodies to CMV. CMV contamination of all investigated blood products, such as platelets, erythrocyte concentrates and fresh frozen plasma, could be proved by PCR analysis. Quantitative PCR analysis determined viral loads up to $10 \mathrm{E} 03$ $\mathrm{IU} / \mathrm{ml}$ in plasma products.

Despite leukocyte-reduction Cytomegalovirus could be detected in blood products. CMV-NAT screening is a strategy to identify viraemic donors and consequently contaminated blood products.

\section{References:}

1 Richtlinien zur Gewinnung von Blut und Blutbestandteilen und zur Anwendung von Blutprodukten (Hämotherapie) Bundesanzeiger Nr. 101a

2 Roth et al (2010) Vox Sang 99 Suppl. 1: 276.

3 Ziemann et al (2007) Transfusion 47: 1972-82.
Prevalence and Incidence of IgG Antibodies against Hepatitis E Virus (HEV) in Blood Donors in Germany Juhl D. ${ }^{1}$, Baylis S. A. ${ }^{2}$, Blümel J. ${ }^{2}$, Görg S. ${ }^{1}$, Glessing P. ${ }^{1}$, Hennig $\mathrm{H}^{1}$

${ }^{1}$ University Hospital of Schleswig-Holstein, Institute of Transfusion Medicine, Lübeck, Deutschland

${ }^{2}$ Paul-Ehrlich-Institut, Federal Institute for Vaccines and Biomedicines, Langen, Deutschland

Background: Hepatitis E virus (HEV) can be transmitted by transfusion, but data on the seroprevalence and incidence of HEV in German blood donors are sparse. For better assessment of the relevance of HEV in transfusion medicine in Germany, a study on the prevalence and incidence of anti-HEV IgG in our blood donor population was performed.

Methods: Samples taken from 1019 randomly selected whole blood donors were tested for anti-HEV IgG by ELISA. Repeatedly reactive results were confirmed to be positive by Western blot. For those donors testing positive, archive samples obtained approximately two years earlier (median), were investigated by ELISA to assess the incidence of anti-HEV IgG.

Results / Conclusions: 69/1019 (6.8\%) donors tested positive for anti-HEV $\mathrm{IgG}$. The prevalence in younger donors (aged between 18 and $40 \mathrm{ys}, 16 / 481$ $[3.3 \%]$ tested positive) was significantly lower compared to the older donors (aged between 41 and 70 ys, 53/538 [9.9\%] positive, $\mathrm{p}<0.0001$ ). No major differences in the prevalence existed between male (43/591 [7.3\%] positive) and female donors $(26 / 428[6.1 \%]$ positive, $\mathrm{p}=0.451)$ and between first-time (3/117 [2.6\%] positive) and regular blood donors (66/902 [7.3\%] positive, $\mathrm{p}=0.054$ ). As 7/69 anti-HEV IgG positive donors tested negative for anti$\mathrm{HEV} \mathrm{IgG} \mathrm{two} \mathrm{years} \mathrm{before,} \mathrm{the} \mathrm{incidence} \mathrm{of} \mathrm{anti-HEV} \mathrm{IgG} \mathrm{was} \mathrm{calculated} \mathrm{to}$ be $0.35 \%$ per year.

The prevalence of anti-HEV IgG in German blood donors is comparable to other European countries; new infections seemed to occur with a magnitude of $0.35 \%$ per year. To obtain more data about the infectivity and the duration of viremia of the newly infected donors, archive samples will be investigated using HEV NAT.

\section{Prevalence of Hepatitis E Virus RNA and Antibodies in German Blood Donors}

Dreier J. ${ }^{1}$, Vollmer T. ${ }^{1}$, Diekmann J. ${ }^{1}$, Johne R. ${ }^{2}$, Eberhardt M. ${ }^{3}$, Knabbe $C^{1}$

${ }^{1}$ Herz- und Diabeteszentrum NRW, Institut für Laboratoriums- und Transfusionsmedizin, Bad Oeynhausen, Deutschland ${ }^{2}$ Bundesinstitut für Risikobewertung, Berlin, Deutschland ${ }^{3}$ TMD Gesellschaft für transfusionsmedizinische Dienste mbH, Kassel, Deutschland

Background: Hepatitis E infection (HEV) in Europe is supposed to nonepidemic, however a considerable high prevalence rate of anti-HEV was found in blood donors of various European countries. The risk of transfusion-transmitted HEV infections by contaminated blood products currently remains unknown, amongst others possibly due to the absence of available sensitive NAT-screening methods.

Methods: From July to September 2011, a total of 41,325 allogenic blood donations from 16,125 individual donors were routinely screened for HEVRNA using the RealStar HEV RT-PCR assay. Particular HEV genotypes of RNA-positive donors were analyzed and the presence of HEV-specific IgM and IgG titers was determined. Furthermore, HEV RNA and HEV-specific IgM and IgG titers were determined in 136 blood donors with elevated ALT levels and 200 donors with non-elevated ALT values.

Results / Conclusions: The sensitivity of the NAT-assay was determined to a $95 \%$ LOD of $4.66 \mathrm{IU} / \mathrm{ml}$. Thirteen donors were HEV RNA positive $(0.08 \%)$, of these only three donors already showed reactive IgM and/or IgG antibody titers (IgM+/IgG-, IgM+/IgG + ). The detected HEV strains all belonged to genotype 3 and were most closely related to German HEV strains from wild boars and pigs as well as from human hepatitis E cases. HEV RNA was not detectable in donors with and without elevated ALT levels, but $8.08 \%$ of donors (elevated ALT) respectively $0.5 \%$ of donors (non-elevated ALT) showed reactive HEV-IgM titers. The overall seroprevalence rate of HEV-IgG accounted to 5.94\% (5.88\% elevated ALT, $6.0 \%$ nonelevated ALT).

Although a considerable high seroprevalence rate and a high number of HEV-RNA positive blood products occurred, the clinical relevance of transfusion-associated hepatitis $\mathrm{E}$ infection still require further investigations. However in case of raising concerns regarding blood safety, we evaluated a sensitive NAT-screening method for the detection of HEV in blood components, providing a sensitive possibility for additional viral testing. 


\section{Pilot Study of Hepatitis E Virus Detection in Blood Donors Using a Multiplex Minipool NAT Assay on the Zelos x100 Platform}

Hourfar M. K., Schmidt M., Sireis W., Capalbo G., Seifried E. DRK Blutspendedienst Baden-Württemberg - Hessen gGmbH, Frankfurt am Main, Deutschland

Introduction: Several transfusion transmitted hepatitis E infections have been reported, although the major infection pathway is via the fecal oral route. Previous studies from UK, Germany, Sweden and Japan demonstrated prevalence in a range between 1 in 4,000 and 1 in 10,000.

Background: The current study reports on the development of a real-time multiplex nucleic acid amplification test (NAT) for blood donor screening on the Zelos x100 platform (automated NAT system developed by the German Red Cross Baden-Württemberg - Hessen). In addition first routine blood screening data from a pilot study including approximately 12,200 blood donations are presented.

Methods: A new multiplex minipool real-time NAT system was developed for the combined amplification and detection of hepatitis E virus, Parvovirus B19 and hepatitis A virus in a multiplex/multicolour system. To identify the incidence of Hepatitis E-virus infections in our blood donor population the NAT assay was used on pools of up to 94 donations per pool.

Results / Conclusions: The combination of our extraction system which is based on a high volume magnetic particle extraction process and the new multiplex PCR allows highly sensitive detection of Hepatitis A-Virus, Parvovirus B19 and Hepatitis E-Virus. First results of the pilot study show that 4 blood donations out of 12,200 were HEV-RNA positive.

The results of blood donor testing demonstrate a comparably high incidence of approximately 1 in 3,000, which is significantly higher than for Hepatitis A-virus. Additionally virus-load in HEV-NAT-positive donations was higher than usually observed for HAV. Therefore it may be recommendable to introduce HEV minipool NAT into blood donor screening.

\section{Progress of Asymptomatically Hepatitis E Infection in German Blood Donors and Comparison of Two Immunological Anti-HEV Tests}

\section{Vollmer $T^{1}{ }^{1}$, Diekmann $J^{1}{ }^{1}$, Johne $R^{2}{ }^{2}$, Eberhardt $M^{3}$,} Knabbe C. ${ }^{1}$, Dreier J. ${ }^{1}$

${ }^{1}$ Herz- und Diabeteszentrum NRW, Institut für Laboratoriums- und Transfusionsmedizin, Bad Oeynhausen, Deutschland ${ }^{2}$ Bundesinstitut für Risikobewertung, Berlin, Deutschland

${ }^{3}$ TMD Gesellschaft für transfusionsmedizinische Dienste mbH, Kassel, Deutschland

Background: Hepatitis E infection (HEV) in Europe is supposed to nonepidemic, but asymptomatic infection was found in blood donors of various European countries. In the present study, we analyzed the progression of HEV RNA concentration, serostatus and liver-specific enzymes in ten German blood donors with asymptomatic HEV infection.

Methods: HEV RNA concentrations were quantified using the first WHO international Standard for hepatitis E Virus RNA for NAT-based assays. Plasma concentrations of alanine/aspartate aminotransferase (ALT/AST) glutamate dehydrogenase (GLDH) and total bilirubin were measured using enzymatic assays. The presence of anti-HEV IgM and IgG antibodies was determined using the different immunological assays recomLine HEVIgM/IgG immunoassay, the MP Bio HEV IgM ELISA 3.0 and MP Bio HEV IgG ELISA and the MP Bio HEV ELISA 4.0 all antibodies.

Results / Conclusions: Observed RNA concentrations ranged from 77.2 to $2.19 \mathrm{E}+05 \mathrm{IU} / \mathrm{ml}$. High virus concentrations were observed combined with a negative HEV serostatus. Nine donors showed a typical progression of a recent HEV infection, however only four of these donor showed elevated ALT, AST and GLDH values. One donor had apparently a chronic HEV infection. Comparison of the different immunological assays was performed on the basis of this ten different seroconversion panels, showing differences regarding the sensitivity and performance between the different assays.

The sensitivity of different anti-HEV immunoassays vary, however, with regards to identification of asymptomatic HEV infected blood donors, an effective screening for HEV infection could be performed only by use of NAT-screening methods.

\section{Bacterial Screening of Platelet Concentrates Using the BactiFlow-ALS-System}

Schottstedt V. ${ }^{1}$, Walther-Wenke G. ${ }^{2}$, Krzos M. ${ }^{1}$, Unnerstall B. ${ }^{2}$, Bux J. ${ }^{1}$

${ }^{1}$ DRK Blutspendedienst West, Zentrallabor Hagen, Deutschland

${ }^{2}$ DRK Blutspendedienst West, Zentrum für Transfusionsmedizin Münster, Deutschland

Introduction: Bacterial sepsis related to transfusion of contaminated platelet concentrates (PC) still is a significant risk of morbidity and mortality. Due to growth behavior of bacteria serious and fatal transfusion reactions tend to occur towards the end of shelf life of PCs [1].

Background: To detect potentially dangerous PCs we introduced a screening on bacteria for all PCs that are in the inventory on day 3 of their shelf life. The screening process has been validated in interlaboratory studies before [2].

Methods: Aliquots of $15 \mathrm{ml}$ out of PCs are drawn into sample bags using sterile docking device. After transportation at ambient temperature from 5 production sites to the central laboratory located in Hagen, testing is performed by using an automated BactiFlow-ALS-system (AES Chemunex/BioMérieux) according to Dreier et al and manufacture instructions [3]. An inactive fluorescent dye is added to the sample that diffuses into bacteria, if present. Bacterial esterase transforms it into a fluorescent dye and count of bacteria is determined by flow cytometry.

Results / Conclusions: Until now a total of 9002 PCs (23\% apheresis PCs, $77 \%$ PCs from four pooled buffy coats) were tested. Within 4 hours one technician is able to perform testing on 160 samples using 3 BactiFlow-ALSsystem. The rate of initially positive results that could not be confirmed showed to be $0.5 \%(n=49)$. Two PCs could be identified as contaminated with S.epidermidis $(183,577$ germs $/ \mathrm{ml})$ and S. capitis $(6,302$ germs $/ \mathrm{ml})$. These PCs were blocked and transfusion could be prevented.

The BactiFlow-system appears to be a fast and robust method to screen large numbers of PCs for bacteria on day 3 of shelf life. Further studies have to prove if this bacterial screening system is capable to remove all bacterially contaminated PCs from the supply and so eliminates transmission of bacteria by PCs on day 4 and 5 of shelf life.

References

1 Funk MB, Lohmann A, Guenay S et al. Transfusion-Transmitted Bacterial Infections - Haemovigilance Data of German Blood Establishments (1997-2010). Transfus Med Hemother 38;2011:266-271.

2 Vollmer T, Hinse D, Schottstedt V et al. Inter-laboratory comparison of different rapid methods for $t$ he detection of bacterial contamination in platelet concentrates. Vox Sang 2011:DOI: 10.1111/j.1423-0410.2011.01572.x.

3 Vollmer T, Engemann J, Kleesiek K et al. Bacterial screening by flow cytometry offers potential for extension of platelet storage: results of 14 month of active surveillance. Transf Med 2011;21:175-182.

\section{Evaluation of the New German Blood Donor Questionnaire}

Sümnig A. ${ }^{1}$, Weber $H^{2}{ }^{2}$, Lembcke $H^{2}{ }^{2}$, Greffin $K^{2}{ }^{2}$, Deitenbeck $R^{3}$, Bux J. ${ }^{3}$, Greinacher A. ${ }^{1}$

${ }^{1}$ Institut für Transfusionsmedizin und Immunologie, Transfusionsmedizin, Greifswald, Deutschland

${ }^{2}$ Institut für Psychologie, Differentielle und Persönlichkeitspsychologie, Greifswald, Deutschland

${ }^{3}$ DRK West, Zentrum für Transfusionsmedizin Hagen, Deutschland

Background: Each blood service has to apply a donor history questionnaire (DHQ), fulfilling the requirements of the German Hemotherapy Guidelines. In February 2011 a uniform blood donor history questionnaire for Germany was provided by the Paul Ehrlich Institute (PEI). The new questionnaire is characterized by non-medical language, easy to understand sentence structure, and specific assessment of sexual behavior. Especially the open wording in regard to sexual behavior raised major concerns about acceptance by donors. We conducted a questionnaire-based study to evaluate perception of the DHQ by blood donors.

Methods: The Department of Transfusion Medicine at the University Hospital in Greifswald and the collection site of the German Red Cross Blood Service West in Hagen implemented the new DHQ and asked donors to fill in a questionnaire evaluating the design, clarity and intimacy of the questions of new DHQ over a time period of two months.

Results / Conclusions: In Greifswald 2000 donors (mean age $32 \pm 12$ years) participated and in Hagen 2088 donors (mean age $45 \pm 14$ years) participated. The new DHQ was regarded to be understandable and clearly arranged. In regard to the questions addressing sexual behavior, perception differed 
significantly depending on donor characteristics: women, donors $<30$ years of age, and donors with school education $>10$ years perceived these questions as more reasonable and important to evaluate risk situations compared to men, donors $>30$ years of age, and donors with school education $<10$ years . Overall, the new DHQ did not significantly impair the willingness of blood donation. The new DHQ was sufficiently accepted in two different study populations. Educational advertising is needed to increase the acceptance in older, male, and less educated donors.

\section{Results of the Usage of a New Standardized Questionnaire in Mobile Blood Collection}

Deitenbeck R. ${ }^{1}$, Alt $T^{2}{ }^{2}$

${ }^{1}$ DRK Blutspendedienst West, Zentrum für Transfusionsmedizin Hagen, Deutschland

${ }^{2}$ DRK Blutspendedienst Rheinland-Pfalz/Saarland, Zentrum für

Transfusionsmedizin Bad Kreuznach, Deutschland

Background: In 2/2011 a new standardized questionnaire (NQ) was recommended by the national authorities to be used in Germany accompanied by a field study for evaluation of it's effects on the supply situation.

Methods: From May 16th 2011 until May 15th 2012 (examination period, EP) the NQ is used by some of our collection teams. Beyond an interim valuation of the key issues of the field study (change of deferral rate (DR) with focus on sexual risks) we examined the compliance with regard to spontaneously expressed complaints.

Results: During the EP the NQ was used by 49.674 donors. The old questionnaire (OQ) was used by 59.569 donors within a comparative period (CP) from 2010 to 2011. Total DR increased by 2.0 percentage points (PP $(p<0.001)$. With recurrent donors (RD) DR increased by $1.7 \mathrm{PP}(\mathrm{p}<0.001)$ and fell by 4.3 PP with new donors (ND) $(\mathrm{p}<0.001)$. With ND DR was $7.39 \%$ regarding the question of unprotected sexual intercourse with a new partner $(0.43 \%$ with $\mathrm{RD})$. Regarding the question of other sexual risks DR with RD increased by $0.0124 \%$ and dropped by $0.64 \%$ with ND. There was no influence of the questionnaire on the confidential self exclusion (CSE) $(+0.01 \%)$. The amount of documented complaints was constantly at about $0.12 \%$ with far more complaints from elderly men $(72.4 \%, \varnothing$ age $51.1 \mathrm{yrs}$.) with numerous donations (Ø 48.8) than from other groups. Nevertheless, $69.0 \%$ of the complainants visit a donation campaign again.

Conclusions: The total DR increases significantly by $2.0 \mathrm{PP}$. The NQ has no influence on the CSE. The donor compliance is much lower with RD compared to other groups (higher complaint rate), though most of the complainants return to one of the next donation campaigns. Actually there is no evidence if the total return rate changes or if the safety of blood products can be raised, since it is unclear if donors give correct information regarding the critical questions. The study will be continued to gain information about the long-term effects regarding behavior of donors.

\section{Developments in Demography and Donation Activity of German Blood and Plasma Donors}

\section{Ritter S. , Hamouda O. , Offergeld R.}

Robert Koch Institute, Department for Infectious Disease Epidemiology, Berlin, Deutschland

Background: The demographic change in Germany is characterized by an aging and shrinking population. While the demand for blood products increases, the number of Germans eligible to donate blood is on the decline since 2003. Detailed knowledge of the demographic profile and donation behavior of donor populations are essential for planning an adequate blood supply.

Methods: Surveillance data from the national epidemiological reporting system and population data from the federal statistical office were used to assess changes in demography and donation frequencies of whole blood and apheresis donors and the percentage of donors among the general population over a 5 year period.

Results / Conclusions: From 2006 to 2010, the general population aged 18 to 68 decreased by $2 \%$. At the same time, the number of first time and repeat whole blood donors rose by $8 \%$ and $7 \%$, respectively. The increase was even greater among plasma donors (23\% for first time and $41 \%$ for repeat donors). In $2010,4.3 \%$ of the population eligible to donate were active as repeat whole blood donors; $0.4 \%$ repeatedly donated plasma or platelets. The age structure of whole blood repeat donors resembled that of the general population, the greatest number of donors being 45 to 54 years of age in 2010 . In contrast nearly $40 \%$ of the repeat plasma donors were younger than 25 years. As observed in previous years, donation frequency increased with age in both sexes. The average for whole blood donations remained unchanged since 2006 at 1.9 per year; for apheresis donations it increased from 11.9 to 12.5 (plasma) and from 4.0 to 5.0 donations (platelets).

The receding number of potential blood donors in Germany in the past years has not led to a decrease in number of donors and donations. By recruiting new and retaining existing ones, the proportion of donors among the general population increased especially for young and older donors. The data presented can be used to assess the consequences of measures that affect the number of donors and/or donations.

\section{New Methods for Detection of Red Blood Cell Antibodies}

Seltsam A.

German Red Cross, Blood Service NSTOB, Springe, Germany

Background: Since red blood cells (RBCs) carry large numbers of antigens, antibody identification in RBC-based assays relies on the lack of reaction of an antibody with panel cells negative for the corresponding antigen. This indirect method of antibody identification is always challenged when a person's serum contains multiple antibodies, autoantibodies or antibodies directed against high-prevalence antigens. The availability of recombinant blood group proteins (rBGPs) makes it possible to develop new antibody detection assays based on the use of a defined antigen for each reaction.

Methods: Blood group proteins were recombinantly expressed in bacteria or eukaryotic cell cultures. The rBGPs were used in soluble protein reagents in inhibition assays or attached to various surfaces for use in other common antibody identification systems such as ELISA, capture technique and colorcoded microspheres. A panel of commercial and patient serum samples containing antibodies against common or high-prevalence antigens was tested. The recombinant proteins were stored for up to 2 years at $4{ }^{\circ} \mathrm{C}$ until use

Results: Single rBGP specificities carrying common (e.g. K, Fya, S) or highprevalence antigens (e.g. Kpb, Lub, Kna) and cocktails containing a mixture of soluble rBGPs were used to identify RBC antibodies. Assays using rBGPs were highly specific and the rBGP-based solid phase assays showed a higher sensitivity than the conventional RBC-based antibody detection methods. Single rBGPs or protein cocktails were used in antibody identification and crossmatching to neutralize antibodies against high-prevalence antigens, allowing for easy detection of underlying antibodies.

Conclusion: Soluble rBGP are stable and can easily be integrated in routine $\mathrm{RBC}$ serology. This novel approach allows direct detection and identification of blood group antibodies in a single step without the need for timeconsuming additional examinations. In addition, it would significantly facilitate automated interpretation of antibody identification test results in routine diagnostics.

\section{Crossmatch versus Type-and-Screen}

Taune-Wikman A.

Immunhaematology Unit, Dept of Clinical Immunology and Transfusion Medicine, Karolinska University Hospital, Stockholm, Sweden

Background: The pretransfusion compatibility testing can be performed by conventional cross-match against every single red cell unit or by type-andscreen and electronic crossmatch. The latter routine has been used in nonimmunized patients in Sweden for the last twenty years.

Methods: In Sweden with approximately 460.000 red cell transfusions per year type-and-screen and electronic crossmatch was introduced in the beginning of the 1990ies. Type-and-screen includes ABO control and screening against 3-4 in-house test cells expressing clinically relevant antigens, in homozygous or heterozygous form, according to national guidelines. If the screening is negative $\mathrm{ABO}$ and $\mathrm{RhD}$ compatible blood units can be issued via an electronic control in the blood data system. The negative screening result is valid for 3-4 days, after that a new type-and-screen is performed. If the screening is positive, antibodies are identified and units negative for corresponding antigen are crossmatched.

Results: The advantages with type-and-screen and electronic crossmatch compared to crossmatch of every single unit is that it saves time, blood can be issued directly without preordering of blood units and focus can be put on those with positive screening results where blood units are selected for crossmatch. The return of issued blood units is low and the wastage of blood can be minimized. The disadvantage with type-and-screen is that antibodies against private antigens not represented in the screening cells could be missed. A weak immune antibody not detected in the screening, could be boostered and thus not detected within 3-4 days. Follow-up data from the 
Swedish national hemovigilance register and from the Karolinska University Laboratory in Stockholm will be presented.

Discussion: After 20 years' experience of type-and-screen and electronic crossmatch the conclusion is that it is safe and time-saving, provided a laboratory information system designed for transfusion medicine is available. The process of pretransfusion testing involves many steps; identity control and blood sampling procedures, request for blood and receipt of referrals and blood samples, criteria for acceptance, procedures for the test methods and the issue of blood. All of these steps are important in issuing the right blood to the right patient with high safety.

\section{Comparison of Quality Standards for ATMPs, Medicinal Products and Tissues and Cells}

\section{Kurz J.}

Bundesministerium für Gesundheit III/4 - Strategische Angelegenheiten, Blut, Gewebe und Transplantationswesen, Wien, Österreich

Introduction: EU Regulation 1394/2007 on Advanced Therapy Medicinal Products (ATMP Reg) defines that somatic cell therapy, gene therapy and tissue engineering products require a central marketing authorisation in the European Union.

Background: Tissue engineered products include tissues and cells, which were subjected to substantial manipulation. ATMP Reg Annex 1, in particular, lists manipulations which shall not be considered as substantial manipulations. These tissues and cells will not require a marketing authorisation and are regulated by the EU Directive 2004/23/EC

In Article 28 of ATMP Reg conditions are defined which are known as "hospital exemption“. According to Article 3 of the medicinal products directive 2001/83/EC the tissues and cells classified as Article 28 products are excluded from Directive 2001/83/EC. Hence it is the responsibility of each EU member state to decide which quality standards should be followed for the manufacturing and pharmacovigilance of these tissues and cells.

Results / Conclusions: Implementing ATMP Reg some stakeholders emphasize the difficulties following quality standards of medicinal products which have been established in the pharmaceutical industry for decades because of claimed high demands on resources for environmental conditions, control, documentation and personnel. These requirements are assumed not to be transferrable to tissues and cells in a hospital setting. On the other hand there are reservations that the exemption of tissues and cells from high medicinal products standards might jeopardise the patients' safety and the quality of products.

In this presentation a direct comparison shall demonstrate the equivalency of tissue and cells and medicinal products regulations highlighting the common goal to achieve patients' safety and quality of products.

\section{References}

Regulation (EC) No 1394/2007 of the European Parliament and of the Council of 13 November 2007 on advanced therapy medicinal products and amending Directive 2001/83/EC and Regulation (EC) No 726/2004. Official Journal L 324/121, $10 / 12 / 2007$.

Directive 2001/83/EC of the European Parliament and of the Council of 6 November 2001 on the Community Code Relating to Medicinal Products for Human Use Official Journal L $-311,28 / 11 / 2004$, p. 67-128 as amended by

Directive 2002/98/EC of the European Parliament and of the Council of 27 January 2003 setting standards of quality and safety for the collection, testing, processing, storage and distribution of human blood and blood components and amending Directive 2001/83/EC. Official Journal L - 33, 08/02/2003, p. 30-40 Directive 2004/24/EC of the European Parliament and the Council of 31 March 2004 amending, as regards traditional herbal medicinal products, Directive 2001/83/EC on the Community code relating to medicinal products for human use. Official Journal L - 136, 30/04/2004, p. 85-90

Directive 2004/27/EC of the European Parliament and the Council of 31 March 2004 amending Directive 2001/83/EC on the Community code relating to medicinal products for human use. Official Journal L - 136, 30/04/2004, p. 34-57.
Commission Directive 2003/94/EC of 8 October 2003 laying down the principles and guidelines of good manufacturing practice in respect of medicinal products for human use and investigational medicinal products for human use. Official Journal L 262/22, $14 / 10 / 2003$

EudraLex - Volume 4 Good manufacturing practice (GMP) Guidelines. http://ec.europa.eu/health/documents/eudralex/vol-4/index en.htm.

Directive 2004/23/EC of the European Parliament and of the Council of 31 March 2004 on setting standards of quality and safety for the donation, procurement, testing, processing, preservation, storage and distribution of human tissues and cells. Official Journal L $102,07 / 04 / 2004$ P. 0048-0058.

Commission Directive 2006/17/EC of 8 February 2006 implementing Directive 2004/23/EC of the European Parliament and of the Council as regards certain technical requirements for the donation, procurement and testing of human tissues and cells. Official Journal L 038, 09/02/2006 P. 0040-0052.

Commission Directive 2006/86/EC of 24 October 2006 implementing Directive 2004/23/EC of the European Parliament and of the Council as regards traceability requirements, notification of serious adverse reactions and events and certain technical requirements for the coding, processing, preservation, storage and distribution of human tissues and cells. Official Journal L 294/32, 25/10/2006.

\section{Feasibility and Safety of Granulocyte Apheresis after G- CSF Stimulation: Final Results of the German Multicenter Trial}

Sachs $U^{1}{ }^{1}$, Bux J. ${ }^{2}$

${ }^{1}$ Justus Liebig-Universität Gießen, Institut für Klinische Immunologie und Transfusionsmedizin, Gießen, Deutschland ${ }^{2}$ DRK Blutspendedienst West, Hagen, Deutschland

Introduction: Report on behalf of all members of the study group. Infections in profound neutropenia after chemotherapy are associated with high mortality despite appropriate antibacterial and antifungal treatment. Granulocyte transfusions (GTX) can be used as a therapeutic addendum. Methods: In a prospective multicenter study encompassing 14 hospitals, feasibility, relevant influence factors, and donor safety aspects were investigated over a study period of 7 years. Non-related community donors were stimulated with G-CSF prior to apheresis. HES 6\%, MW $450 \mathrm{kDa}$ was used during the procedure.

Results / Conclusions: 415 different donors were enrolled. Out of 1,115 requested GTX, 1,107 could be produced and transfused to patients. Multivariate analysis of influence factors on stimulation success and apheresis success revealed no differences with regard to donor sex or type of G-CSF used. Stimulation success was found to be predictive for the apheresis success. Repeated donations in a series led to higher success rates regardless of G-CSF type or donor sex. Relevant side effects included ostealgia, headache, muscle pain, and fatigue. Side effects were more often in females. The frequency of side effects was not correlated with G-CSF dosage or stimulation success. There was a tendency towards fewer side effects in donors over 40 years of age. Multiple donations in a series did not have an effect on the frequency of side effects. Follow-up examination of donors revealed mild changes in blood tests or subjective well-being in 36/291 donors (itching, 5 donors) after 4 weeks and in 24/171 donors after two years (itching, 3 donors). Severe side effects after GTX were not reported for all 1,107 transfusions in this study.

In conclusion, it is feasible and safe to provide granulocyte concentrates after G-CSF stimulation. Repeated donations in a series (every second day) are a reasonable strategy to provide granulocyte concentrates since the granulocyte content increases with the number of donations, whereas side effects do not. 